\title{
Médiévales
}

Langues, Textes, Histoire

66 | printemps 2014

Harmonie Disharmonie

\section{Didier LETT, Hommes et Femmes au Moyen Âge. Histoire} du genre. $X I I^{e}-X V^{e}$ siècle

Paris, Armand Colin («Cursus Histoire »), 2013, 222 p.

Yasmina Foehr-Janssens

\section{CpenEdition}

1 Journals

\section{Édition électronique}

URL : https://journals.openedition.org/medievales/7306

DOI : 10.4000/medievales.7306

ISSN : $1777-5892$

Éditeur

Presses universitaires de Vincennes

Édition imprimée

Date de publication : 30 juin 2014

Pagination : 212-215

ISBN : 978-2-84292-405-8

ISSN : 0751-2708

Référence électronique

Yasmina Foehr-Janssens, " Didier LETT, Hommes et Femmes au Moyen Âge. Histoire du genre. $x{ }^{e}{ }^{e}-x{ }^{e}$ siècle », Médiévales [En ligne], 66 | printemps 2014, mis en ligne le 10 juillet 2014, consulté le 22 avril 2022. URL : http://journals.openedition.org/medievales/7306 ; DOI : https://doi.org/10.4000/ medievales.7306

Ce document a été généré automatiquement le 22 avril 2022.

Tous droits réservés 


\title{
Didier LETT, Hommes et Femmes au Moyen Âge. Histoire du genre. XII ${ }^{e}-X V^{e}$ siècle
}

Paris, Armand Colin («Cursus Histoire »), 2013, 222 p.

\author{
Yasmina Foehr-Janssens
}

\section{RÉFÉRENCE}

Didier LETT, Hommes et Femmes au Moyen Âge. Histoire du genre. XII ${ }^{e}-\mathrm{XV}^{e}$ siècle, Paris, Armand Colin («Cursus Histoire »), 2013, 222 p.

1 Consacrer à l'histoire du genre au Moyen Âge un volume d'une collection de manuels universitaires n'est pas un geste anodin. Il faut donc saluer cette initiative bienvenue qui confère une légitimité nouvelle à une perspective critique longtemps restée marginale dans le monde académique français et considérée avec suspicion comme un "produit d'importation", selon la belle formule de D. Lett (p. 9). Le concept de genre forgé dans le sillage de la critique féministe a pris racine dans de très nombreuses disciplines de sciences humaines aux États-Unis, ainsi que dans les mondes anglo-saxon et germanique dès les années 1970. Sa diffusion dans la réflexion académique francophone a été plus lente et il est remarquable que sa relative généralisation coïncide, sur la scène politique, avec de multiples interventions destinées à dénoncer dans « la théorie du genre » une idéologie qui nierait la différence des sexes.

2 Outil conceptuel bien plus que corps de doctrine, la notion de genre sert de levier à de très nombreuses études concernant les rôles sociaux de sexe. Elle est donc parfaitement adaptée aux recherches historiques, dès lors que celles-ci envisagent leurs objets en s'interrogeant sur l'impact différencié que ceux-ci peuvent avoir sur les hommes et sur les femmes dans un état de société donné. C'est ce que démontre très clairement $\mathrm{D}$. Lett en envisageant «l'agencement particulier des rapports de sexes » (p. 10) au Moyen Âge dans des contextes factuels ou discursifs variés. Les données rassemblées dans cet 
ouvrage concernent aussi bien l'histoire littéraire que les études de démographie, la médecine que la théologie, l'anthroponymie et la sigillographie que la pédagogie médiévales, sans parler bien entendu du droit, des théories politiques, de la philosophie morale et des données fournies par des analyses économiques. L'histoire du genre au Moyen Âge, considérée à partir de points de vue aussi variés, révèle une cartographie complexe des relations entre hommes et femmes. Impossible de nier que la domination masculine s'exprime tant dans la lecture des textes bibliques que dans les statuts juridiques. Assignation des femmes à la sphère privée et définition des statuts légaux et sociaux de celles-ci en fonction de leurs positions à l'égard de l'institution matrimoniale sont de règle. Cependant, la prise en compte de sources documentaires non normatives permet de nuancer le tableau, de repérer des espaces ou des marges d'autonomie que peut conférer l'appartenance à certaines catégories sociales ou maritales (haute noblesse, artisanat urbain, veuvage) et de mettre en évidence des évolutions, comme celle que connaît la régence confiée à une femme (p. 144-146).

3 Le grand mérite de l'ouvrage de D. Lett tient dans son approche nuancée, dans sa rigueur méthodologique et dans l'ampleur de sa documentation. Loin de s'en tenir à une synthèse des études menées depuis plus de trente ans dans le domaine de l'histoire des femmes, il nous offre un panorama incluant les travaux sur les masculinités médiévales. Structuré en trois grandes parties centrées successivement sur les identités, la culture et la société, l'exposé envisage tout d'abord les « fondements de la distinction des sexes " reposant sur les interprétations du récit de la Création, la conception médicale des corps sexués, ainsi que les statuts sociaux et leurs marques identificatoires (noms, sceaux, vêtements). L'examen de l'éducation différenciée, des rapports à l'institution ecclésiastique, des croyances et des pratiques religieuses, du rapport aux pouvoirs juridique et politique forme la seconde partie. Le travail, la violence et la sexualité font l'objet de la troisième partie. La présentation de résultats alterne avec la discussion des thèses les plus importantes dans le champ de l'histoire du genre, comme (aux pages 30 à 32) celles de Thomas Laqueur sur les deux modèles concurrents de conception de la différence des sexes (La Fabrique du sexe, 1990, trad. française 1992). De même, lorsqu'il commente, en référence aux travaux de l'anthropologue P. Tabet ( $\mathrm{La}$ Construction sociale de l'inégalité des sexes. Des outils et des corps, 1998), la « domination masculine par l'outil» (p. 155-157) et signale la répartition genrée des instruments de production dans les métiers de la paysannerie, D. Lett met son lecteur en garde contre la tentation d'essentialiser ces dichotomies culturelles si profondément ancrées qu'elles peuvent facilement passer pour des données « naturelles ».

4 Au-delà de la mise en évidence de polarités clairement établies, les études portant sur le genre s'intéressent aussi au brouillage des dichotomies, aux phénomènes d'hybridation ou de renversement entre les valeurs du féminin et du masculin. Là encore, D. Lett rend compte avec clarté de l'état des recherches. La tentation est grande, à cet égard, d'interpréter tout écart par rapport à la norme d'une stricte ségrégation des valeurs, des pratiques et des catégories de genre comme le symptôme d'une subversion possible du modèle dominant. C'est un travers dans lequel l'auteur se garde bien de donner. Ainsi la présentation des modèles de masculinité s'attache à rappeler qu'à partir du XII ${ }^{e}$ siècle, sous l'influence de la réforme grégorienne, l'état clérical se doit d'affirmer sa précellence par la valorisation de la maîtrise de soi, de sorte que le renoncement à la sexualité se construit comme l'affirmation d'une virilité supérieure et non d'une quelconque féminisation (p. 48-49). Par contre, la 
disqualification de catégories sociales et religieuses infériorisées (juifs, mendiants ou autre marginaux) passe, quant à elle, par l'attribution de caractères culturellement marqués par le féminin (p. 54). Cependant, le jeu sur les catégories peut se faire plus complexe, comme dans le cas de la communauté universitaire, caractérisée à la fois par les excès et les turbulences de la jeunesse mâle, par un statut social clérical imposant une forme d'identité asexuée et par une identification à une communauté conçue sur un mode symbolique comme une entité féminine, l'alma mater (p. 51-52). Dans ce cas, troublant pour une perception moderne, il convient, pour comprendre une telle construction sociale, d'accepter de se déprendre de modes de pensée marqués par les dichotomies contemporaines. De même, le paragraphe consacré aux pratiques de travestissement s'accompagne d'une mise au point méthodologique qui insiste sur une nécessaire contextualisation (p. 73-74).

On doit donc à D. Lett un ouvrage d'une grande valeur heuristique, reposant sur une très vaste documentation et une connaissance approfondie des dossiers traités. S'il fallait exprimer un regret, ce serait celui de voir le souci de rigueur méthodologique prendre le pas sur la reconnaissance de certaines audaces de la pensée médiévale. Certes, le motif de l'allaitement masculin, spirituel ou miraculeux (p. 125-126), que l'auteur connaît bien pour avoir donné des travaux importants sur ce sujet ${ }^{1}$, est à comprendre comme un fantasme masculin dans un contexte dominé par la métaphore collective du sein de l'Église et par une constante inquiétude à l'égard de la survie des nouveaux-nés. Il n'en demeure pas moins que l'image saisissante de Claire d'Assise tétant le sein de saint François relève d'une puissance d'invention symbolique qui traduit, de la part des religieuses mystiques, une capacité à se saisir du répertoire des représentations théologiques et à le remodeler au gré de leurs propres expériences, tant spirituelles qu'existentielles. À force de resituer les expressions les plus originales du système du genre au Moyen Âge dans le contexte d'un monde régi par la pensée patriarcale, on manque peut-être l'occasion d'interroger les moyens qui s'offrent aux groupes dominés, et notamment aux femmes, d'exercer leur capacité d'action dans un univers de pensée masculin. Cette agency, selon le terme consacré en anglais, se manifeste avec insistance dans le champ artistique, par exemple dans les œuvres des femmes de lettres. Comme le souligne D. Lett, celles qui nous sont connues appartiennent à un cercle restreint, d'où émerge une poignée de noms : Hildegarde de Bingen, Clemence de Barking, Marie de France, Catherine de Sutton, les trobaitritz Marie de Ventadour ou Na Castelloza, Marguerite Porete et, bien sûr, Christine de Pizan. Cependant, outre le fait que l'anonymat assez généralisé de la production poétique en langue vernaculaire laisse dans l'ombre l'appartenance à un sexe ou à l'autre de la grande majorité des rédacteurs ou rédactrices de ces textes anciens, l'examen des œuvres que nous ont laissées les femmes auteures révèle la hardiesse de leur pensée et leur capacité à contrer, par l'ironie le plus souvent, les préjugés défavorables à leur sexe. C'est pourquoi le choix, certes parfaitement compréhensible sur le plan de la méthode, qu'a fait $\mathrm{D}$. Lett de consacrer le tout premier paragraphe de son livre à asseoir l'idée qu'Héloïse, Hildegarde de Bingen et Christine de Pizan « sont citées à tort comme les premières féministes de notre histoire » (p.9), me paraît pouvoir être discuté2. Bien entendu, il serait vain de vouloir rabattre les revendications d'égalité politique et de libération sexuelle des mouvements féministes des $\mathrm{XIX}^{\mathrm{e}}$ et $\mathrm{XX}^{\mathrm{e}}$ siècles sur les conceptions $\mathrm{du}$ monde et de la vie en société des intellectuelles $d u \mathrm{XII}^{\mathrm{e}}$ ou $\mathrm{du} \mathrm{XV}^{\mathrm{e}}$ siècle. Il n'en demeure pas moins que la pensée politique de Christine de Pizan, la puissance visionnaire d'Hildegarde ou le génie poétique de Marie de France ne sauraient être 
réduits à leur apparente adhésion aux valeurs de leur temps. Par bien des aspects, ils témoignent au contraire d'une très claire conscience de la violence des rapports sociaux de sexe et d'une analyse subtile des mécanismes de la domination. Christine, pour ne citer qu'elle, peut sembler acquiescer au dogme de la supériorité masculine, mais c'est le plus souvent pour retourner les armes de l'adversaire contre lui. Elle semble avoir parfaitement compris, avant la lettre, les enjeux symboliques de la " domination par l'outil ». Avec un art consommé, elle confisque à son propre usage les emblèmes virils de la pioche, de l'épée et de la plume pour se construire un éthos de " fille d'étude » toujours prête à "se dresser » pour reprendre le travail grâce à « l'outil de son intelligence $»^{3}$. Si elle feint de se lamenter, au début de la Cité des Dames, sur son incapacité féminine ${ }^{4}$, c'est pour mieux jeter le ridicule sur l'arrogance et les déviances du discours misogyne : Dieu aurait-il commis une erreur en créant la femme ${ }^{5}$ ? S'il y a peut-être quelque anachronisme terminologique à qualifier de féministe un tel discours, on ne saurait cependant nier qu'il ne le cède en rien, en fait d'assurance et d'ambitions intellectuelles, au féminisme contemporain.

\section{NOTES}

1. D. LETT, «L'allaitement des saints au Moyen Âge. Un seul sein vénérable : le sein de la Vierge », dans D. BONNET, C. LE GRAND-SEBILLE, M.-F. MOREL éd., Allaitements en marge, Paris, 2002, p. 163-174; D. LETT et M.-F. MOREL, Une histoire de l'allaitement, Paris, 2006.

2. À propos de ce débat, on peut signaler la parution d'un recueil de textes réunis par $\mathrm{N}$. PELLEGRIN éd., Écrits féministes de Christine de Pizan à Simone de Beauvoir, Paris, 2010.

3. CHRISTINE DE PIZAN, Le Livre des trois vertus, éd. C. C. WILLARD et E. HICKS, Paris, 1989, p. 7-8, 1. 15-28.

4. Voir la citation choisie par Didier Lett, p. 9.

5. CHRISTINE DE PIZAN, Le Livre de la Cité des Dames, trad. par E. HICKS et T. MOREAU, Paris, 1986 (« Moyen Âge »), p. 37 : "Ah! Seigneur ! [...] comment croire, sans tomber dans l'erreur, que ton infinie sagesse ait pu créer quelque chose qui ne soit pas entièrement bon?» 